\title{
The Genomic Landscape of Mucinous Breast Cancer
}

\author{
Fresia Pareja*, Ju Youn Lee*, David N. Brown, Salvatore Piscuoglio, Rodrigo Gularte-Mérida, \\ Pier Selenica, Arnaud Da Cruz Paula, Sasi Arunachalam, Rahul Kumar, Felipe C. Geyer, \\ Catarina Silveira, Edaise M. da Silva, Anqi Li, Caterina Marchiò, Charlotte K. Y. Ng, \\ Odette Mariani, Laetitia Fuhrmann, Hannah Y. Wen, Larry Norton, Anne Vincent-Salomon, \\ Edi Brogi, Jorge S. Reis-Filho, Britta Weigelt
}

*Authors contributed equally to this work.

Correspondence to: Jorge S. Reis-Filho, MD, PhD, FRCPath, Department of Pathology, Memorial Sloan Kettering Cancer Center, 1275 York Avenue, New York, NY 10065 (e-mail: reisfilj@mskcc.org) and Britta Weigelt, PhD, Department of Pathology, Memorial Sloan Kettering Cancer Center, 1275 York Avenue, New York, NY 10065 (e-mail: weigeltb@mskcc.org).

\begin{abstract}
Mucinous carcinoma of the breast (MCB) is a rare histologic form of estrogen receptor (ER)-positive/HER2-negative breast cancer $(\mathrm{BC})$ characterized by tumor cells floating in lakes of mucin. We assessed the genomic landscape of $32 \mathrm{MCBs}$ by wholeexome sequencing and/or RNA-sequencing. GATA3 (23.8\%), KMT2C (19.0\%), and MAP3K1 (14.3\%) were the most frequently mutated genes in pure MCBs. In addition, two recurrent but not pathognomonic fusion genes, OAZ1-CSNK1G2 and RFC4-LPP, were detected in 3/31 (9.7\%) and 2/31 (6.5\%) samples, respectively. Compared with ER-positive/HER2-negative common forms of BC, MCBs displayed lower PIK3CA and TP53 mutation rates and fewer concurrent 1q gains and 16q losses. Clonal decomposition analysis of the mucinous and ductal components independently microdissected from five mixed MCBs revealed that they are clonally related and evolve following clonal selection or parallel evolution. Our findings indicate that MCB represents a genetically distinct ER-positive/HER2-negative form of BC.
\end{abstract}

Mucinous carcinoma of the breast (MCB) is a rare histologic type of estrogen receptor (ER)-positive/HER2-negative breast cancer (BC) characterized by tumor cells floating in mucin $(1,2)$. Pure MCBs (PMCBs) display more than $90 \%$ of mucinous areas, are associated with a favorable clinical outcome (3), and can be subclassified into type A (paucicellular) and type B (hypercellular) (4). Mixed MCBs (MMCBs) contain $50-90 \%$ of mucinous areas (1) and portend a worse outcome than PMCBs (5).

Despite their unique phenotype and previous efforts to characterize their genomic landscape by copy number and targeted sequencing analyses $(6,7)$, no pathognomonic genetic alteration underpinning PMCBs or the mucinous phenotype have been identified. Hence, we sought to characterize the repertoire of somatic genetic alterations of MCBs by whole-exome sequencing (WES), to determine their differences from ER-positive/HER2negative invasive ductal carcinomas of no special type (IDCNSTs) and invasive lobular carcinomas (ILCs), and to determine whether they would be underpinned by highly recurrent fusion genes. The study was approved by the local ethics committees of the authors' institutions, and informed consents were obtained. The methods employed for WES and RNA-sequencing and for data analyses are detailed in the Supplementary Methods (available online). Statistical significance was evaluated by the two-sided Mann-Whitney $U$ test and Fisher's exact test for continuous and categorical variables, respectively. $P$ values less than .05 were considered statistically significant.

Thirty-two MCBs, of which 25 were PMCBs (13 type A and 12 type B) and seven MMCBs, were included in this study (Supplementary Table 1, available online). Following microdissection, 28 MCBs were subjected to WES and 15 MCBs to RNAsequencing (Supplementary Tables 1 and 2, available online). GATA3 (23.8\%), KMT2C (19.0\%), and MAP3K1 (14.3\%) were the most frequently mutated genes in PMCBs (Figure 1A). All GATA3 mutations were frameshifting, and $80.0 \%$ (4 of 5) were clonal 
A
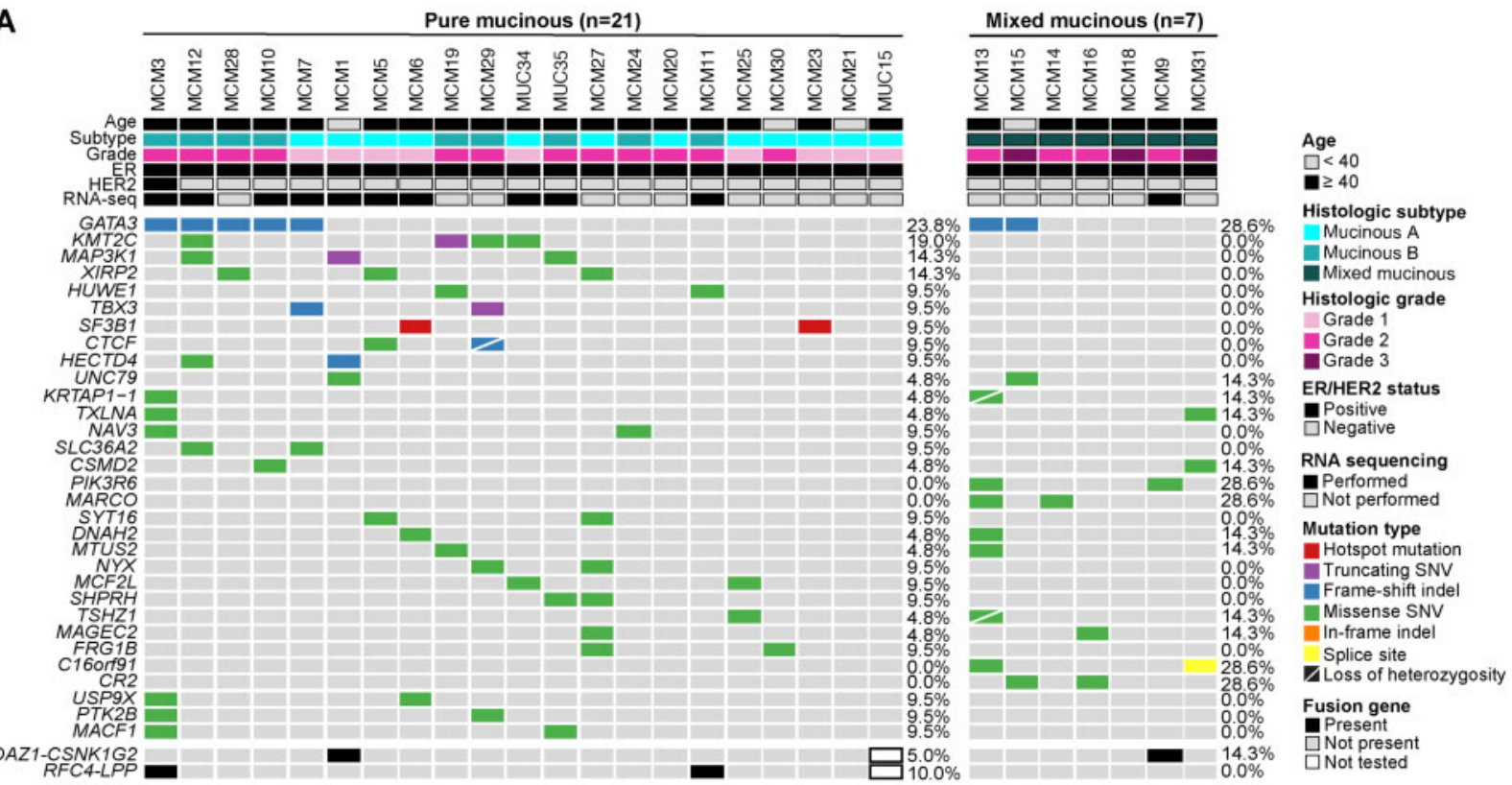

B
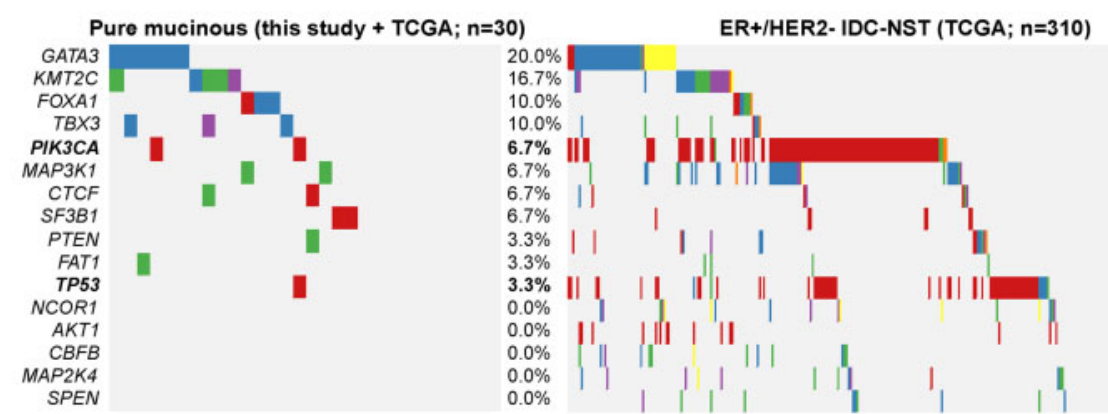

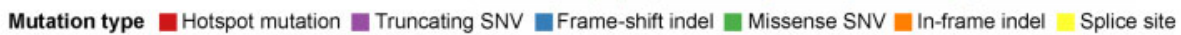

$\begin{array}{ll} & \boldsymbol{P} \text { value } \\ 1 \% & 0.61 \\ 0 \% & 0.34 \\ \% & 0.08 \\ \% & 0.06 \\ .4 \% & <0.001 \\ 6 \% & 0.55 \\ \% & 0.18 \\ \% & 0.18 \\ \% & 0.99 \\ \% & 0.37 \\ 0 \% & 0.02 \\ \% & 0.38 \\ \% & 0.38 \\ \% & 0.62 \\ \% & 0.99\end{array}$

C

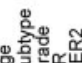

D
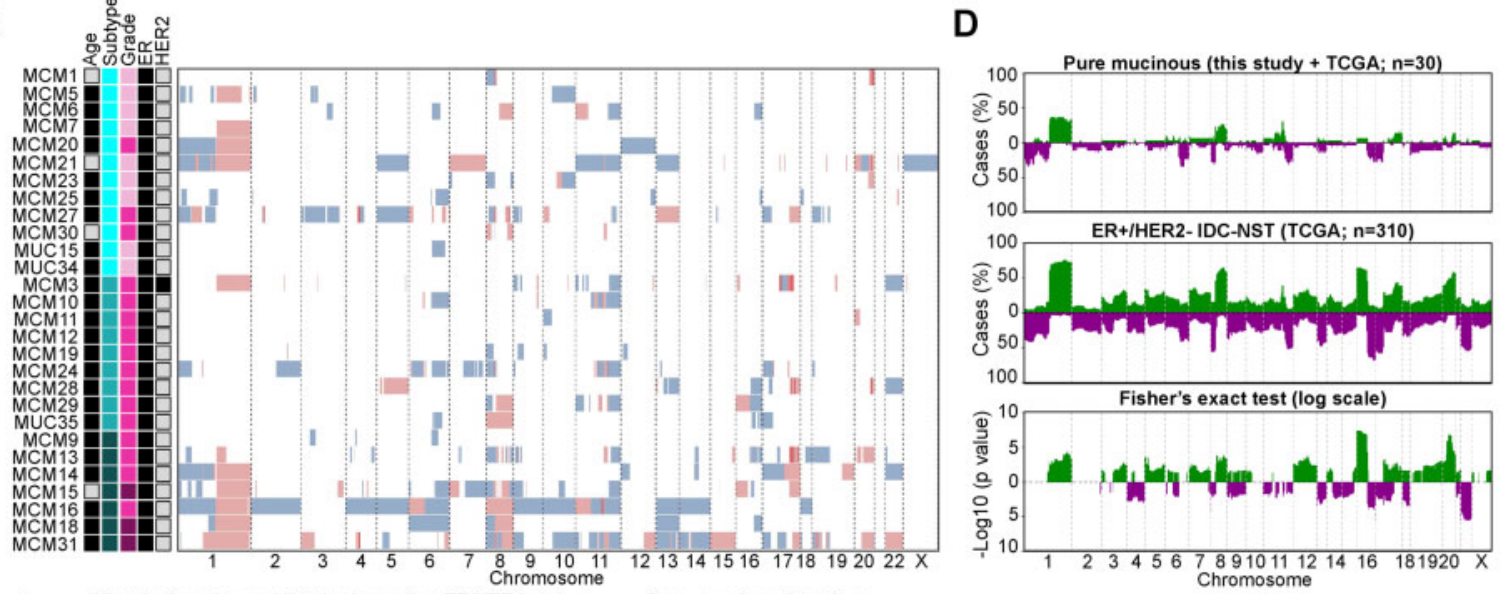

Age Histologic subtype Histologic grade ER/HER2 status

Copy number alterations

$\square<40$ Mucinous A Grade 1 - Positive Amplification Homozygous deletion

Mucinous B Grade 2

Figure 1. Landscape of somatic genetic alterations in mucinous breast cancers. A) Recurrent somatic mutations and fusion genes identified in pure mucinous carcinomas of the breast (PMCBs; $\mathrm{n}=21$; left) and in the mucinous component of mixed mucinous carcinomas (MMCBs; $\mathrm{n}=7$; right), subjected to whole-exome sequencing (WES; $\mathrm{n}=28$ ) and/or to RNA-sequencing (RNA-seq; $\mathrm{n}=11$ ). Cases are shown in columns and genes in rows. Histopathologic characteristics are depicted in the phenotype bars (top). Genetic alterations are color-coded according to the legend, and loss of heterozygosity is represented by a diagonal bar. B) Comparison of the most frequently mutated cancer genes identified in estrogen receptor (ER)-positive/HER2-negative PMCBs from this study ( $\mathrm{n}=20$ ) and from The Cancer Genome Atlas (TCGA; $\mathrm{n}=10$; total $\mathrm{n}=30$ ) and in ER-positive/HER2-negative invasive ductal carcinomas of no special type (IDC-NSTs) from TCGA ( $=310$ ). Cases are shown in columns and genes in rows. Fisher's exact test. C) Copy number alterations identified in PMCBs $(n=21)$ and in the mucinous component of MMCBs ( $\mathrm{n}=7$ ) subjected to WES. Cases are depicted in rows and chromosomes along the $x$-axis. Histopathologic characteristics are indicated (left) and color-coded according to the legend. Dark red, amplification; light red, copy number gain; dark blue, homozygous deletion; light blue, copy number loss; white, copy neutral. D) Frequency plots and Fisher's exact test 
(Supplementary Figure 1A, available online). In addition, two PMCBs harbored a clonal SF3B1 K700E hotspot mutation. Importantly, PMCBs displayed a low frequency of mutations affecting PIK3CA (4.8\%) or TP53 (4.8\%), which are frequently found in ER-positive BCs (Figure 1, A and B; Supplementary Table 3, available online) (8). An exploratory analysis of the mutational repertoire in type A and type B PMCBs revealed no statistically significant differences (Supplementary Table 4, available online). Of the cases harboring sufficient mutations to infer their mutational signatures, 4/5 (80.0\%) PMCBs displayed a dominant signature 1 (aging) and one (20.0\%) a dominant signature 2 (APOBEC) (9), and 2/3 MMCBs displayed a dominant signature 1 in both the mucinous and ductal components (Supplementary Figure 1, B and C, available online). Consistent with previous reports $(6,10)$, none of the MCBs analyzed here harbored concurrent $1 \mathrm{q}$ whole-arm gains and $16 \mathrm{q}$ whole-arm losses, a hallmark of ER-positive/HER2-negative BCs (11) (Figure 1C).

ER-positive/HER2-negative PMCBs (this study and The Cancer Genome Atlas [TCGA], $\mathrm{n}=30$ ) had a lower tumor mutation burden (TMB) than the mucinous component of MMCBs, but similar TMB to ER-positive/HER2-negative IDCs-NSTs and ILCs from TCGA (8) (Supplementary Figure 1, D and E, available online). Despite the similar TMBs, mutations affecting PIK3CA and TP53 were statistically significantly less frequently $(P<.001$, two-sided Fisher's exact test) detected in PMCBs (this study and TCGA, $\mathrm{n}=30$; this study and METABRIC, $\mathrm{n}=56$ ) than in all ERpositive/HER2-negative IDCs-NSTs from TCGA $(\mathrm{n}=310)$ and METABRIC $(\mathrm{n}=977)(8,12,13)$ and in IDC-NSTs matched according to age and/or menopausal status (TCGA, $n=90$; METABRIC, $\mathrm{n}=168$; Figure $1 \mathrm{~B}$ and Supplementary Figure 2, A-H, available online). A comparison of ER-positive/HER2-negative PMCBs $(\mathrm{n}=30)$ with ER-positive/HER2-negative ILCs from TCGA (all, $\mathrm{n}=73$; menopausal status-matched, $\mathrm{n}=60$ ) and METABRIC (all, $\mathrm{n}=117$; menopausal status-matched, $\mathrm{n}=56$ ) revealed a statistically significantly lower frequency of mutations targeting PIK3CA and CDH1 $(P<.001$, two-sided Fisher's exact test; Supplementary Figure 2, A-H, available online). No statistically significant differences in the frequency and spectrum of GATA3 mutations were observed between ER-positive/HER2-negative PMCBs and ER-positive/HER2-negative ILCs and IDC-NSTs. It should be noted, however, that at variance with PMCBs, which almost exclusively harbored GATA3 frame shift mutations, GATA3 mutations in ILCs and IDC-NSTs comprised missense, frameshift, truncating, and splice-site mutations (Supplementary Figure 2, I-R, available online). Reanalysis of the gene copy number alterations found in ER-positive/HER2negative PMCBs from this study $(n=20)$, TCGA $(n=10)$, and METABRIC $(n=36)$ revealed statistically significantly less frequent gains of $1 \mathrm{q}$ and $16 \mathrm{p}$ and losses of $16 \mathrm{q}$ and $22 \mathrm{q}$ than in all and age- and/or menopausal status-matched ER-positive/HER2negative IDC-NSTs (TCGA, $\mathrm{n}=310$ and $\mathrm{n}=90$; METABRIC, $\mathrm{n}=977$ and $\mathrm{n}=168$ ) and ILCs (TCGA, $\mathrm{n}=73$ and $\mathrm{n}=60$ ) (Figure 1D; Supplementary Figure 3, A-J, available online). Copy number analyses at the gene level confirmed these observations (Supplementary Figure 3, K-M, available online). In addition, ERpositive/HER2-negative PMCBs from our study $(n=12)$ displayed higher expression levels of mucin-encoding genes, including MUC2 and MUC4, than ER-positive/HER2-negative IDC-NSTs (all, $\mathrm{n}=310$; age- and menopausal status-matched, $\mathrm{n}=36$ ) and ILCs (all, $\mathrm{n}=73$; menopausal status-matched, $\mathrm{n}=24$ ) from TCGA (Supplementary Figure 4, A and B, available online). Taken together, the statistically significantly lower frequencies of PIK3CA mutations, $1 \mathrm{q}$ gains and $16 \mathrm{q}$ losses in PMCBs are consistent with the notion that these tumors may follow an evolutionary pathway distinct from that of the archetypal ER-positive BC.

RNA-sequencing analysis of six type $A$ and eight type $B$ PMCBs and the mucinous component of a MMCB revealed no pathognomonic fusion gene but resulted in the identification of fusion genes present in more than one sample in $28.6 \%(4 / 14)$ of PMCBs and in the sole MMCB analyzed (Supplementary Figure 4C; Supplementary Table 5, available online). Of these, orthogonally validated likely pathogenic fusion genes included OAZ1-CSNK1G2 and RFC4-LPP, which were detected in 3/31 (9.7\%) and $2 / 31(6.5 \%)$ of all cases interrogated, respectively. OAZ1-CSNK1G2 results in the fusion of exon 1 of OAZ1 with exons 9-11 of CSNK1G2, which causes the deletion of the serine threonine kinase region of the latter that is required for repression of ER transactivation, suggesting a potential role for this fusion in ER transactivation. The RFC4-LPP chimeric transcript results from the fusion of exons 1-3 of RFC4 with exons 5-11 of the known fusion gene partner LPP, which is reported to mediate TGF- $\beta$-induced breast oncogenesis $(14,15)$. Additional fusion genes identified in MCBs were IRAK3-PPM1H $(n=1)$, GIGYF2GFRA3 $(\mathrm{n}=1)$, and PHF20-FAM217B $(\mathrm{n}=1)$, most of which involved kinases, phosphatases, or regulators of tyrosine kinase receptor signaling (16-19) (Supplementary Figure 4D, available online).

As an exploratory, hypothesis-generating analysis to identify genes specific to the mucinous phenotype, we subjected independently microdissected mucinous and ductal components from five MMCBs to WES (Supplementary Methods; Supplementary Table 2, available online). This analysis did not reveal any highly recurrently mutated (ie, in more than two of five cases) genes but demonstrated that the mucinous and ductal components of MMCBs are heterogeneous and clonally related (Figure 2, A-C; Supplementary Figure 4, E and F, available online). In three MMCBs, minor subclones of the mucinous components harboring mutations affecting GATA3, LPP or NF1 became dominant in the ductal component, likely following clonal selection (Figure 2, A-C). In two MMCBs, the mucinous and ductal components appeared to have undergone parallel evolution (Supplementary Figure 4, E and F, available online). Although most PMCBs and MMCBs displayed an aging signature (ie, signature 1), MCM15, which arose in a BRCA1 germline pathogenic mutation (p.C16G) carrier whose tumor also harbored somatic loss of heterozygosity of the BRCA1 wild-type allele in both histologic components (ie, BRCA1 bi-allelic inactivation), displayed the homologous recombination DNA repair deficiency-related mutational signature 3 (Figure 2B; Supplementary Figures $1 \mathrm{C}$ and $4, \mathrm{G}$ and $\mathrm{H}$, available online). Taken together, these findings suggest that the mucinous and ductal components of MMCBs are clonally related, and, at least in a subset of cases, the ductal component likely stemmed from the mucinous areas.

Our study has important limitations, such as the small sample size, given the rarity of MCBs. In addition, given the multi-

Figure 1. Continued

corrected for multiple testing comparing copy number gains and losses between the combined cohort of ER-positive/HER2-negative PMCBs from this study ( $\mathrm{n}=20$ ) and TCGA ( $n=10$; total $n=30)$ and ER-positive/HER2-negative IDC-NSTs from TCGA $(n=310)$. The frequency of gains (green bars) or losses (purple bars) for each gene is plotted on the $y$-axis according to genomic position ( $x$-axis). Inverse Log $_{10}$ values of the Fisher's exact test $P$ values are plotted according to genomic location (lower panel). All statistical tests were two-sided. indel = small insertion/deletion; SNV = single nucleotide variant. 
A
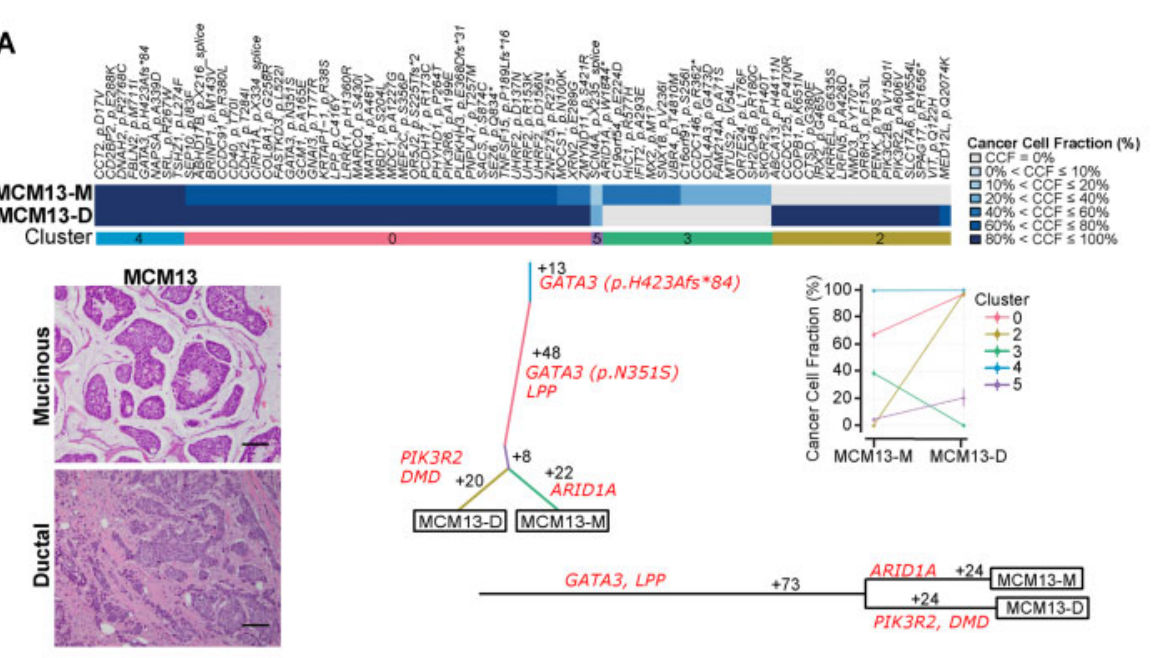

MCM13-D MCM13-M

B

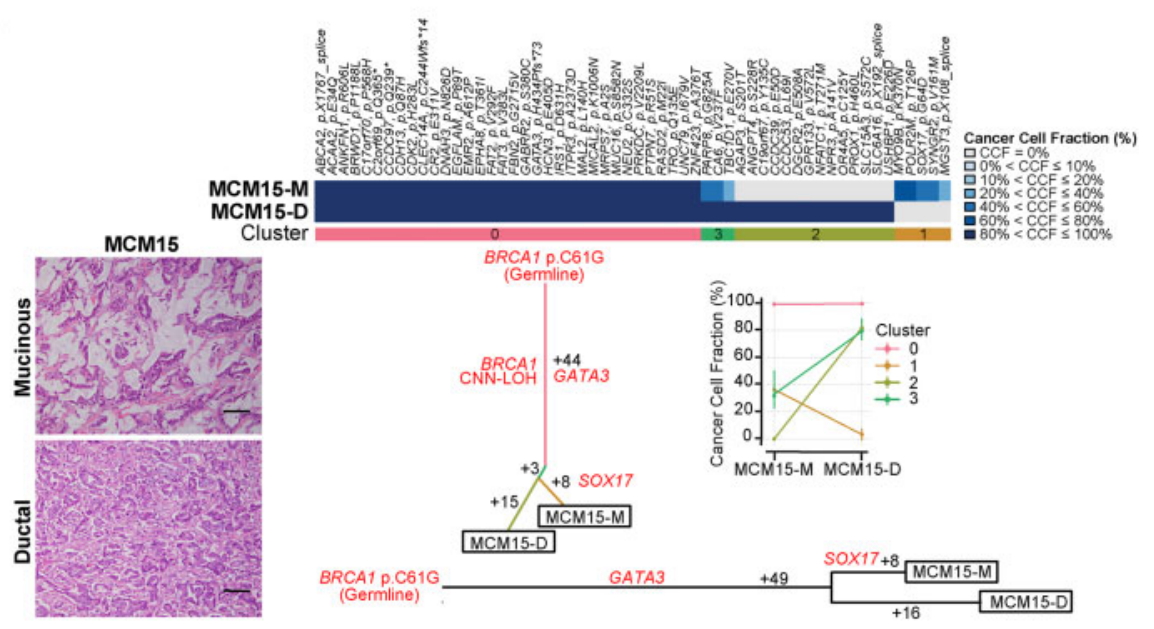

C

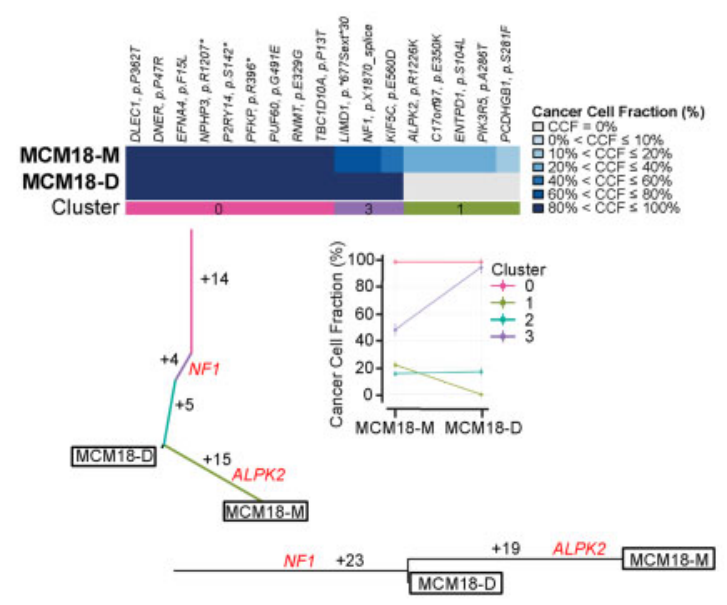

Figure 2. Clonal composition of the mucinous and ductal components of mixed mucinous breast cancers (MMCBs). Representative hematoxylin-and-eosin micrographs of the mucinous and ductal histologic components of MMCBs (A) MCM13, (B) MCM15, and (C) MCM18 (left). Clonal frequency heatmaps of mutations in the mucinous (M) and ductal (D) histologic components of a given MMCB are shown (top), grouped by their clonal/subclonal structure (clusters) as inferred by PyClone (see Supplementary Methods). Cancer cell fractions are color-coded according to the legend (top right). PyClone clusters are shown in the bottom of the clonal frequency heatmap. A cluster-based phylogenetic tree of MMCBs (middle center) and a parallel coordinates plot generated by PyClone (middle right) are shown. Trunk and branches are colored according to clusters as per PyClone, and the number of somatic mutations that result in the divergence of a clone/subclone from its ancestor are shown. Cancer genes are depicted in red. Phylogenetic trees generated using Treeomics (see Supplementary Methods) are depicted (bottom). The length of the trunk and branches represent the number of shared and private somatic mutations identified in both histologic components, respectively. Scale bar, $20 \mu \mathrm{m}$. 
institutional nature of our study, survival analyses could not be performed. Nonetheless, our findings demonstrate that MCBs are genetically heterogeneous and lack a pathognomonic fusion gene or somatic mutation, but differ from common forms of ERpositive/HER2-negative BCs, based on the remarkably low frequency of 1q gains, 16q losses, and PIK3CA and TP53 mutations.

\section{Funding}

This work was supported by the Breast Cancer Research Foundation and a Cancer Center Support Grant of the National Institutes of Health/National Cancer Institute (grant no. P30CA008748). The content is solely the responsibility of the authors and does not necessarily represent the official views of the National Institutes of Health. Salvatore Piscuoglio was supported by a Susan G. Komen Research and Training Grant (PDF14298348). Caterina Marchiò is supported in part by FPRC$5 \times 1000$ MIUR.

Jorge S. Reis-Filho reports personal/consultancy fees from VolitionRx, Page.AI, Goldman Sachs, Grail, Ventana Medical Systems, Roche, Genentech and Invicro, outside the scope of the submitted work. The remaining authors declare no competing financial interest. The funders of this study had no role in the design of the study; the collection, analysis, and interpretation of the data; the writing of the manuscript; and the decision to submit the manuscript for publication.

\section{References}

1. Lakhani SR, Ellis IO, Schnitt SJ, et al. WHO Classification of Breast Tumors. Lyon IARC; 2012.

2. Harrison BT, Dillon DA. An update of mucinous lesions of the breast. Surg Pathol Clin. 2018;11(1):61-90.

3. Cao AY, He M, Liu ZB, et al. Outcome of pure mucinous breast carcinoma compared to infiltrating ductal carcinoma: a population-based study from China. Ann Surg Oncol. 2012;19(9):3019-3027.
4. Capella C, Eusebi V, Mann B, et al. Endocrine differentiation in mucoid carcinoma of the breast. Histopathology. 1980;4(6):613-630.

5. Skotnicki P, Sas-Korczynska B, Strzepek L, et al. Pure and mixed mucinous carcinoma of the breast: a comparison of clinical outcomes and treatment results. Breast J. 2016;22(5):529-534.

6. Lacroix-Triki M, Suarez PH, MacKay A, et al. Mucinous carcinoma of the breast is genomically distinct from invasive ductal carcinomas of no special type. J Pathol. 2010;222(3):282-298.

7. Kehr EL, Jorns JM, Ang D, et al. Mucinous breast carcinomas lack PIK3CA and AKT1 mutations. Hum Pathol. 2012;43(12):2207-2212.

8. Cancer Genome Atlas Network. Comprehensive molecular portraits of human breast tumours. Nature. 2012;490(7418):61-70.

9. Nik-Zainal S, Morganella S. Mutational signatures in breast cancer: the problem at the DNA level. Clin Cancer Res. 2017;23(11):2617-2629.

10. Mukheriee A, Russell R, Chin SF, et al. Associations between genomic stratification of breast cancer and centrally reviewed tumour pathology in the METABRIC cohort. NPJ Breast Cancer. 2018;4:5.

11. Natrajan R, Lambros MB, Geyer FC, et al. Loss of $16 q$ in high grade breast cancer is associated with estrogen receptor status: Evidence for progression in tumors with a luminal phenotype? Genes Chromosomes Cancer. 2009;48(4): 351-365.

12. Pereira B, Chin SF, Rueda OM, et al. The somatic mutation profiles of 2,433 breast cancers refines their genomic and transcriptomic landscapes. Nat Commun. 2016;7:11479.

13. Curtis C, Shah SP, Chin SF, et al. The genomic and transcriptomic architecture of 2,000 breast tumours reveals novel subgroups. Nature. 2012;486(7403): 346-352.

14. Ngan E, Northey JJ, Brown CM, et al. A complex containing LPP and alphaactinin mediates TGFbeta-induced migration and invasion of ErbB2 expressing breast cancer cells. J Cell Sci. 2013;126(9):1981-1991.

15. Ngan E, Stoletov K, Smith HW, et al. LPP is a Src substrate required for invadopodia formation and efficient breast cancer lung metastasis. Nat Commun 2017;8:15059.

16. Kesselring R, Glaesner J, Hiergeist A, et al. IRAK-M expression in tumor cells supports colorectal cancer progression through reduction of antimicrobial defense and stabilization of STAT3. Cancer Cell. 2016;29(5): 684-696.

17. Shen T, Sun C, Zhang Z, et al. Specific control of BMP signaling and mesenchymal differentiation by cytoplasmic phosphatase PPM1H. Cell Res. 2014 24(6):727-741.

18. Giovannone B, Lee E, Laviola L, et al. Two novel proteins that are linked to insulin-like growth factor (IGF-I) receptors by the Grb10 adapter and modulate IGF-I signaling. J Biol Chem. 2003;278(34):31564-31573.

19. Airaksinen MS, Saarma M. The GDNF family: signalling, biological functions and therapeutic value. Nat Rev Neurosci. 2002;3(5):383-394. 